\title{
La ciencia en los discursos escritos por estudiantes de Bachillerato
}

\section{Science representations in writing compositions by mexican high school students}

\author{
Víctor Gálvez Díaz \\ Secretaría de Educación Pública. Dirección General de Educación Tecnológica Industrial. México \\ galvezvictor@yahoo.com \\ Rosa Nidia Buenfil Burgos \\ Centro de Investigación y Estudios Avanzados. Departamento de Investigaciones Educativas. México \\ rbuenfil@cinvestav.mx
}

RESUMEN • Este informe describe las representaciones de la ciencia expresadas en textos escritos por estudiantes de tres escuelas de bachillerato con características socioculturales diferentes. Para conformar su representación de la ciencia, los estudiantes emplean una variedad de elementos discursivos relacionados con diferentes categorías epistemológicas, como la realidad, la observación y el método científico, entre otros. Se identifican las tendencias en la expresión de estos elementos discursivoepistemológicos y se sugieren posibles interpretaciones. El estudio de las representaciones científicas adquiere relevancia, en tanto que los valores y las creencias relacionadas con la ciencia y su desarrollo influyen en las actuaciones de los sujetos en contextos como el escolar, donde la ciencia se encuentra cotidianamente presente.

PALABRAS CLAVE: representación social de la ciencia; aprendizaje colaborativo; naturaleza de la ciencia; semiología; análisis del discurso.

ABSTRACT - This report describes the notions (representations) of science as expressed in texts written by high school students from three different sociocultural backgrounds. To build their representations of science, students use a wide variety of discursive elements which are related with different epistemological categories such as reality, observation, scientific method, among others. Trends in the expression of these discursive and epistemological elements are identified, and their possible interpretations are suggested. The study of the representations about science becomes highly relevant when the values and beliefs related to science and its development influence the performances of the social actors in contexts such as school, where science is dealt with daily.

KEYWORDS: social representation of science; collaborative learning; nature of science; semiotics; discourse analysis. 


\section{INTRODUCCIÓN}

Cuando se pide a estudiantes de bachillerato que describan qué significa ciencia, escriben textos como el siguiente:

[La ciencia] hace milagros, logra hasta lo inimaginable e incluso alcanza fronteras que son todo menos accesibles [...] como arma, de la misma forma que puede crear los elementos más magníficos, también puede destruirlos con gran facilidad (Érika, 18 ańos, Ciudad de México).

Mediante el uso de elementos discursivos, como las metáforas (“arma”, entidad que hace "milagros"), los adjetivos y las hipérboles ("crear los elementos más magníficos”) y aquellos elementos que permiten interpretar el sentido de un enunciado ("considero", "de acuerdo con mi opinión"), los informantes expresan lo que piensan y sienten acerca de la ciencia. Para describir la ciencia, los sujetos participantes en esta investigación emplean elementos de diferente naturaleza epistemológica; por ejemplo, algunos consideran que para conocer las cosas, los hechos y los fenómenos que existen "dentro y fuera del hombre" es necesario seguir un método único y seriado que inicia con la observación (postura empírico-inductivista). Otros consideran que después de observar las propiedades de los fenómenos se ponen "leyes al resultado de una investigación" (generalización inductiva).

En este trabajo se describen los elementos discursivo-epistemológicos (EDE) con los que nueve estudiantes, de tres escuelas del nivel medio superior, construyen sus representaciones individuales de la ciencia. Los EDE se agruparon a partir de las semejanzas que guardan entre sí y con determinadas posturas epistemológicas, como el realismo, el empirismo o el positivismo. Esta identificación y posterior agrupación de los EDE no tiene intenciones normativas ni prescriptivas; en el contexto de esta investigación permitió identificar las tendencias en la expresión de los EDE entre los sujetos participantes.

\section{MARCO TEÓRICO}

El estudio de las representaciones de la ciencia en contextos educativos involucra la articulación de diversos aportes, como aquellos que se refieren a las representaciones sociales, a la naturaleza de las ciencias o a la semiología, por lo que, a continuación, se presenta una síntesis de cada uno de ellos.

\section{La naturaleza de la ciencia}

En el contexto de los estudios sobre la enseñanza de las ciencias, la noción naturaleza de la ciencia $(\mathrm{NdC})$ alude, desde el punto de vista epistemológico, a la ciencia como una manera de conocimiento y, desde el punto de vista sociológico, a los valores y las creencias inherentes al conocimiento científico y su desarrollo (Lederman, 1992).

La NdC estudia los supuestos epistemológicos que subyacen a los procesos científicos, esto es, a las actividades relacionadas con la colecta y la interpretación de los datos. Por ejemplo, se analiza de qué manera un procedimiento científico -como la observación- se encuentra guiado por las ideas y los conceptos que el sujeto cognoscente posee sobre el objeto o fenómeno observado (Lederman et al., 2002).

\section{Las representaciones sociales}

Se ha investigado que los sujetos interpretan y construyen los elementos que conforman su mundo mediante representaciones, que normalmente son construidas en la interacción con otros. Tal es el 
caso de elementos como el universo (Nascimento-Schulze, 1999), Dios (Lindeman et al., 2002), el psicoanálisis (Moscovici, 1979) o la función de enfermera (Guimelli, 2001).

Una representación social es un sistema complejo e interrelacionado de ideas, nociones y creencias, compartido en diferente grado por varios sujetos. Es la parte de la representación personal de un objeto que tiene su origen en el intercambio social. Facilita la comunicación y el pensamiento cotidianos, transformando los aspectos complejos y extraños en algo familiar (Fife-Schaw, 1993; Lindeman et al., 2002).

Desde una perspectiva estructural, se puede analizar el contenido y la estructura-organización de una representación social (Abric, 2001). A partir de este enfoque teórico-metodológico, una representación social puede ser considerada como un campo estructurado, o bien un núcleo estructurante. En el primer caso, se ponen de manifiesto los constituyentes de las representaciones (informaciones, imágenes, ${ }^{1}$ creencias, valores, opiniones, elementos culturales, ideológicos, actitudes). En el segundo, se analizan las estructuras elementales alrededor de las cuales se organizan los sistemas de representación, de tal manera que sus elementos se encuentran jerarquizados en centrales y periféricos, manteniendo interrelaciones que determinan su significación y su lugar dentro de la representación.

El sistema central es esencialmente social. Es decir: está relacionado con las condiciones históricas, sociológicas e ideológicas en las que se genera; su origen se encuentra en el contexto social que define las normas y los valores de los sujetos y los grupos. Este sistema le brinda estabilidad y coherencia a la representación, por lo que evoluciona en forma muy lenta. El sistema periférico, más flexible que el núcleo central, se encuentra asociado al contexto inmediato y contingente; mediante la integración de nueva información, permite la adaptación y diferenciación de la representación, así como las modulaciones personales en torno al núcleo central común, generando representaciones y prácticas individualizadas.

\section{Las representaciones sociales de la ciencia}

Una representación social de la ciencia (RSC) es considerada en esta investigación como un tipo particular de representación social, que permite a los sujetos o grupos pensar y construir conocimientos acerca de temas científicos que les son desconocidos. Se construyen a partir de conocimiento compartido dentro de una comunidad, en un determinado momento.

En el contexto escolar, las RSC constituyen construcciones particulares de la realidad; son el fruto de diferentes mediaciones institucionales que se llevan a cabo a través de decisiones y discriminaciones, que se realizan a partir de lo que se define como lo válidamente cognoscible, pues definen autorizadamente lo que es el mundo, y que la escuela se encarga de transmitir. Estas decisiones instituyen el conocimiento legítimo (Edwards, 1999).

Las RSC conforman todas las mediaciones curriculares de la enseñanza y del aprendizaje escolares (Quiroz, 1998), que incluyen las intenciones y finalidades generales de los documentos oficiales (programas, acuerdos) (Gálvez, 2002), los materiales educativos (libros de texto, programas de televisión) (Gálvez y Waldegg, 2004) y, finalmente, los maestros/as que transforman estos insumos en prácticas de enseñanza.

Por lo anterior, es de suponer que las RSC se expresen y puedan identificarse en prácticas de aprendizaje desarrolladas cotidianamente por el alumnado del nivel medio superior, como son los diversos materiales escritos que realizan en las asignaturas científicas (ensayos, monografías, resúmenes, reseñas y reportes de práctica, de visita o de investigación).

1. Para Ryder, Leach y Driver (1999) las imágenes de la ciencia son tipos particulares de representaciones sociales, que son generadas a través de la interacción social y tienen una influencia significativa en la acción y en el pensamiento del individuo. 


\section{La perspectiva semiológica}

La construcción, la comunicación y la negociación de significados entre los sujetos se realizan a partir del empleo de signos. Tanto la semiología como la semiótica aluden al análisis de los procesos de sentido que se verifican en sistemas de signos como el lenguaje escrito, la moda o el lenguaje audiovisual. Así, los signos, como las palabras, las imágenes, los sonidos, los gestos o los objetos (como las prendas de vestir), son estudiados como parte de sistemas complejos de significación (Barthes, 1993).

Al igual que el constructivismo semiótico, en esta investigación se considera que el signo establece una relación de sustitución simbólica con la cosa, por lo que el uso de una palabra o una imagen tiene como fin evocar, aludir, vincular o sustituir la cosa en su ausencia. El lenguaje y los signos que lo conforman incorporan las convenciones socioculturales de una comunidad y, al mismo tiempo, desempeñan un papel central en la construcción social de la realidad (Haidar, 1996).

El análisis del discurso, por su parte, es la disciplina que estudia cómo se conforman las significaciones y sus efectos sociales, así como las funciones comunicativas del lenguaje en tanto actividad inserta en un contexto social (Charaudeau y Maingueneau, 2005).

En esta investigación se analizan los discursos escritos por las alumnas y los alumnos desde una perspectiva semiológica, al identificar los signos, su estructura, los sentidos preferentes y las estrategias discursivas usadas para describir las características de la ciencia.

\section{La articulación de aportes teóricos diversos}

A raíz de este entretejido de diversos conceptos y campos disciplinares, así como de la necesidad actual de estudios multirreferenciales o transdiciplinares, valdría la pena mencionar que, en campos de la vida social con una gran complejidad, como es la educación, la imbricación de perspectivas disciplinares forma parte del habitus académico (Buenfil, 2011). No por ello se obvia el cuidado epistemológico requerido para todo constructo teórico que se reconoce a sí mismo como híbrido o, como los griegos señalaban sin connotación peyorativa, ecléctico (Dogan y Pahre, 1993). Esta vigilancia epistemológica incluye la consistencia y compatibilidad de principios, posicionamientos, lógicas y conceptos de los campos que se estén articulando.

\section{METODOLOGÍA}

\section{Contexto de investigación}

Esta investigación se desarrolló en el marco del proyecto Técnicas de aprendizaje colaborativo con tecnologías de información y comunicación en ciencias (TACTICs) -realizado conjuntamente por el Centro de Investigación y de Estudios Avanzados (cinvestav), México, y la Universidad de Montreal, Canadá-, en el que participaron estudiantes (de entre 15 y 18 años) de varias escuelas del nivel medio superior, con características geográficas, sociales y culturales diversas. El objetivo de TACTICs consiste en favorecer la construcción colectiva de conocimientos sobre una temática general, en el contexto de la integración de disciplinas curriculares (física, química, biología, matemáticas, ciencias sociales, español y lenguas extranjeras). El diseńo didáctico del proyecto contempla la formación de equipos que elaboran, intercambian y responden a una serie de preguntas sobre un tema particular, empleando para ello procesadores de texto e imagen, así como software de trabajo colaborativo en red (Waldegg, 2002). 


\section{Estrategia de recolección de datos}

Para la identificación de los EDE y su participación en la constitución de RSC:

1. Se formó un equipo con tres participantes en el proyecto тастісs durante el periodo 20032004, en tres ciudades de la República Mexicana: Ciudad de México (D. F.), Jojutla (Morelos) y Pachuca (Hidalgo). La participación de los estudiantes fue voluntaria; debían realizar las tareas propias de su equipo TAстісs y las diseñadas para esta investigación particular.

2. Teniendo en cuenta que el trabajo realizado en тастісs se basa en el intercambio de imágenes y textos, que se publican en una página web, se pidió a cada participante la redacción de un texto breve para identificar las RSC mediante su análisis semiológico-discursivo. Las instrucciones que se les dieron fueron las siguientes:

Texto individual

1. Escribe un texto donde menciones:

a) Qué es la ciencia (características, propiedades, naturaleza, finalidad).

b) Qué hacen y cómo trabajan los/as científicos/as (actividades, procesos, acciones que realizan).

2. Complementa lo que dices con ejemplos o mediante un relato (cuento, narración).

3. La extensión del texto debe ser de una cuartilla, aproximadamente.

4. Tiempo: una hora.

\section{Estrategia de análisis}

Lo que opinan los estudiantes sobre la ciencia, como una manera de conocer y de desarrollar el conocimiento científico, ha sido una preocupación permanente de los educadores en ciencias. Estos estudios se iniciaron en la década de 1970 y para ello se ha empleado una gran variedad de instrumentos interpretativos, como las entrevistas (Ryder et al., 1999; Newton y Newton, 1998), los mapas conceptuales y las redes semánticas (Ault et al., 1984; Novak, 1990) o los cuestionarios estandarizados (Wilson, 1954; Lederman et al., 2002).

A estos estudios siguieron aquellos que investigaron los contenidos sobre la $\mathrm{NdC}$ en el currículo y las concepciones que de ella tienen las maestras y los maestros (Aguirre et al., 1990; Southerland y Gess-Newsome, 1999). Después de aproximadamente cinco décadas de investigación, puede afirmarse que tanto el alumnado como el profesorado carecen en general de creencias adecuadas sobre la $\mathrm{NdC}$; que estos contenidos pueden aprenderse mejor mediante una enseñanza explícita y reflexiva en diversos contextos de aprendizaje; que las creencias del profesorado no se trasladan necesariamente de manera automática a la práctica docente, sino que este proceso es muy complejo, y, finalmente, que los/as profesores/as no parecen valorar la $\mathrm{NdC}$ como un objetivo educativo, de la misma forma que lo hace con otros contenidos más tradicionales de las asignaturas científicas (Acevedo, 2008).

El análisis de textos e imágenes mediante herramientas propias de la semiología y del análisis del discurso ha resultado fructífero para identificar las RSC presentes en programas de televisión y en textos escritos por estudiantes (Gálvez y Waldegg, 2003, 2004; Gálvez, 2012).

Por lo tanto, de los textos producidos se analizó el contenido de las RSC tomando en cuenta sus componentes: informaciones, imágenes, creencias, valores, opiniones, elementos culturales e ideológicos (Jodelet, 1988), así como la forma en la que se construyen discursivamente. Para ello:

1. Se consideraron los textos escritos por los/as participantes como discursos (Buenfil y Ruiz, 1997), teniendo en cuenta:

- Su dimensión significante, que permite la construcción y apropiación de la realidad. 
- Su significación abierta a partir de su carácter relacional con otros elementos presentes o ausentes (evocados por asociación) en la cadena discursiva.

- Su condición de comunicación de sentido socialmente compartido y accesible.

- La posibilidad de acceder al significado a partir de su relación con otros discursos, dado que el significado depende del uso que se le dé en un contexto específico.

2. Se focalizaron los elementos comunicativo-discursivos utilizados de manera más evidente en cada segmento del texto, tales como:

a) Los procesos de significación empleados: significados denotados, connotados e ideológicos (Barthes, 1992); las figuras de significado o tropos, como las metáforas (Lakoff y Johnson, 1980) e hipérboles; el énfasis (Charaudeau y Maingueneau, 2002), y la iteración (Derrida, 1989; Poloniato, 1998).

b) Las estrategias lingüísticas enunciativas (Buenfil, 1994), a través de las que el/la hablante intenta establecer ciertas construcciones del mundo en su audiencia.

3. Se siguió la secuencia analítica siguiente:

a) Identificación de los EDE presentes en cada fragmento de discurso (ver "Resultados").

b) Segmentación de cada texto de acuerdo con las temáticas desarrolladas por cada alumno/a.

c) Análisis de la forma en que los EDE organizan la significación de cada tema-segmento del discurso.

d) Identificación de la forma en que los EDE organizan la significación de la totalidad del texto, para conformar una representación particular de la ciencia.

e) Agrupamiento de los EDE con los que los/as estudiantes construyen su representación de la ciencia, a partir de las semejanzas que se observan entre ellos y su parecido con alguna postura epistemológica. Esto es, a partir de los enunciados de los/as informantes, se buscaron los referentes teóricos que dan cuenta de las características enunciadas, independientemente de si tales referentes se ubican en el campo filosófico o de la naturaleza de la ciencia. Así, en algunas ocasiones, un diccionario general de filosofía (Ferrater Mora, 1994; Blauberg, 1975) brindó estos referentes y, en otras, se encontraron en debates o monografías (Marcuse, 2003; Zea, 1985).

Este procedimiento difiere de otros que realizan una clasificación previa y los datos se cotejan con las categorías establecidas. ${ }^{2}$ Para asegurar la representatividad de esta agrupación de EDE, se empleó el método de saturación (Callejo, 1998)³ (ver "Confiabilidad de los datos").

\section{RESULTADOS}

\section{Elementos discursivo-epistemológicos}

Existe una gran diversidad de EDE, con los que los/las estudiantes construyen sus representaciones de la ciencia. A continuación, se presentan los agrupamientos en los que se ordenaron los EDE identifi-

2. Uso teoricista: se refiere a la creencia de que "la teoría establece conceptos a los que la realidad debe ajustarse". Este uso derivado de una forma excesiva e irreflexiva del modelo hipotético-deductivo, sostenida además en un racionalismo que no reconocía cuestionamiento alguno y que fue lamentablemente frecuente en los años setenta, hace de las teorías cuerpos fijos, más o menos rígidos de conocimientos, mediante los cuales los fenómenos estudiados son no solo ordenados sino ajustados a la teoría, que opera como una especie de "camisa de fuerza" (Buenfil, 2011:214).

3. "La saturación señala una tarea de control metodológico, interna al proceso de investigación con prácticas cualitativas, referida a la correspondencia y generalización de los resultados, como ocurre en casi todos los tipos de validez” (Callejo, 1998: 94). 
cados, se incluye un fragmento del discurso representativo de cada elemento y se describe la postura epistemológica a la que se asemeja.

\section{La realidad}

[Ciencia] es el estudio de todo lo que existe y sus medios para manifestarse, sus fenómenos y causas (Lucio, Jojutla).

El sentido de este EDE se relaciona con las posturas realistas, que consideran que los objetos de la realidad tienen características propias e independientes de las ideas, creencias o conocimientos de quien los estudia (Moreno y Waldegg, 1998).

\section{Los procesos empíricos}

Los EDE que se agruparon en esta categoría corresponden a características del conocimiento sostenidas por posturas que podrían ubicarse bajo el paraguas del empírico-inductivismo:

a) Énfasis en los procesos

"Uno de los procesos más comunes en el desarrollo de la investigación es: La elección de un tema. El desarrollo de una hipótesis. La selección de su material de trabajo. El procedimiento. La experimentación. El resultado de la hipótesis y el desarrollo de la teoría" (Lucio, Jojutla).

Se refiere a los procesos que caracterizan la forma de trabajar de las personas que se dedican a la ciencia, por ejemplo, a las actividades relacionadas con la colecta y la interpretación de los datos (Millar y Driver, 1987).

b) Énfasis en la experiencia-experimentación

"Pero si tratamos de darle características a lo que la ciencia posee, podrían ser [...] comprobable por el medio experimental..." (Érika, Ciudad de México).

Todo conocimiento deriva de la experiencia sensible y es validado por ella (Ferrater Mora, 1994).

c) Observación

La ciencia es "un proceso que va desde la observación hasta los resultados obtenidos" (Marco, Jojutla).

Se pueden establecer como verdaderos los enunciados hechos acerca del mundo por un observador libre de prejuicios (Chalmers, 1998).

d) Pensamiento lógico

"La ciencia es la que se encarga de saber las cosas y encontrarles una lógica verdadera a los fenómenos ocurridos dentro y fuera del hombre" (Antonia, Pachuca).

Hace referencia a la acepción de la lógica como una forma de pensar y de decir capaz de describir la esencia de las cosas (Ferrater Mora, 1994).

e) Generalización inductiva

"Es necesario observar las [sic] cambios de determinados fenómenos y sus propiedades de cada uno de estos para que así se llegue a una conclusión [...] para poner leyes al resultado de una investigación" (Marco, Jojutla). 
El método empírico de las ciencias naturales consistente en recopilar hechos (a partir de los cuales se formulan enunciados singulares) mediante la observación y la experimentación cuidadosas para, posteriormente, derivar leyes y teorías (expresadas a través enunciados generales) por medio de algún procedimiento lógico como la generalización (Chalmers, 1998).

\section{f) Procedimiento deductivo}

[Los científicos] "realizan diferentes investigaciones para para [sic] desarrollar hipótesis y comprobar teorías que sirvan para el conocimiento personal y universal [generalización inductiva], explicar los fenómenos [explicación deductiva], conocer nuevas cosas, ir más lejos" (Lucio, Jojutla).

El procedimiento inductivo se complementa con otro deductivo en el que, a partir de los enunciados generales, en forma de leyes y teorías, es posible explicar y predecir hechos y fenómenos, expresados en enunciados singulares.

\section{La ciencia es positiva}

El siguiente grupo de EDE, presentes en los textos escritos por los/as informantes, corresponde a rasgos que en general son considerados como de naturaleza positivista:

a) El conocimiento científico es positivo

La ciencia es "un instrumento por medio del cual el hombre puede constatar de manera experimental [...] cualquier objeto de estudio o fenómeno natural, de una manera exacta utilizando una serie de mecanismos e instrumentos por medio de los cuales se puede obtener resultados certeros" (Érika, Ciudad de México).

El conocimiento es positivo cuando es preciso, riguroso, estricto, cierto, efectivo, verdadero y se puede constatar (Ferrater Mora, 1998). Así, el conocimiento científico positivo:

1. Se encuentra verificado-comprobado:

"La ciencia siempre tiene y/o busca la comprobación de los fenómenos estudiados" (Antonia, Pachuca).

Se excluye todo aquello que no puede ser verificado por la observación, por lo que la experiencia es considerada como un conocimiento supremo (Marcuse, 2003).

2. Es sistematizado y ordenado:

Dividir el conocimiento científico en varias fracciones "es provechoso en el sentido de que se vuelve más específico y, de esta manera, las respuestas que se le encuentren serán cada vez más exactas" (Azucena, Ciudad de México).

El conocimiento científico se encuentra sistematizado (ordenado, organizado) en campos de estudio especializados; es "preciso, riguroso, efectivo, verdadero, comprobado" (Ferrater Mora, 1998); y "progresa armoniosamente" (Marcuse, 2003). 


\section{b) Método}

"Las personas que se dedican a la ciencia, es decir los científicos, llevan a cabo investigaciones basándose en un método científico que consta de: Observación [...] Hipótesis [...] Experimentación [...] Postulación de leyes" (Antonia, Pachuca).

Se considera al método científico como un conjunto de reglas perfectamente definidas, ordenadas y seriadas que, si se siguen de forma mecánica, conducen al conocimiento (Nieda y Macedo, 2008).

\section{c) Acumulación de conocimientos}

"Los científicos son las personas que buscan esta ciencia con el fin de ampliar sus conocimientos [...] gracias a ella el hombre puede extender sus conocimientos al máximo [...]" (Fernando, Pachuca).

La ciencia se desarrolla por acumulación de conocimientos, donde cada científico (a) agrega un piso más a los ya consolidados (Nieda y Macedo, 2008).

\section{d) Utilitarismo}

"La Ciencia data de muchos años a tras $[s i c]$ desde que apareció el primer hombre en la Tierra ya que él buscó la manera de sobrevivir [...]” (Gonzalo, Pachuca).

Sugiere que la ciencia positiva se preocupa por el conocimiento útil, antes que por la contemplación ociosa (Marcuse, 2003).

\section{e) Utilitarismo tecnológico}

"Existe demasiada $[s i c]$ relación entre ciencia y tecnología que se llega hasta el punto de que si no existe la ciencia, no existe la tecnología, es decir, 'si no hay cimientos, no hay edificio'" (Fernando, Pachuca).

Se equipara ciencia con tecnología o se considera que la tecnología es únicamente la aplicación de la ciencia que permite mejorar las condiciones de vida del ser humano, por lo tanto, la tecnología nunca es primero (Driver et al., 1989).

f) Actividad solo para iniciados/as

"[...] por ejemplo el origen de la vida un tema donde surgen cuestiones y dudas que no cualquier persona pede $[s i c]$ resolver tan fácilmente ya que para descifrar este tipo de cuestiones hay que [sic] una larga lista de procedimientos que deben llevarse a cabo [...]" (Sonia, Jojutla).

Los sabios poseen el más poderoso de todos los elementos para mejorar las condiciones sociales y para servir dignamente a la humanidad: la ciencia (Macedo en Zea, 1985).

g) Fe ilimitada

"Hoy en día escuchamos hablar constantemente de la Ciencia, cómo ésta hace milagros, logra hasta lo inimaginable e incluso alcanza fronteras que son todo menos accesibles" (Érika, Ciudad de México).

Debido al desarrollo técnico-industrial del siglo xIx, los positivistas creyeron haber encontrado en la ciencia una garantía infalible del destino del ser humano (Abbagnano y Visalberghi, 2008).

h) Significación religiosa positivista

"De acuerdo a mi opinión todo lo que vemos y hasta lo que no vemos podemos decir que es ciencia [...]" (Marco, Pachuca).

La ciencia se exalta y se le considera la única manifestación legítima de lo infinito, cargándose de significación religiosa (Abbagnano y Visalberghi, 2008). 


\section{Elementos metafisicos}

En este agrupamiento se incluyen aquellos EDE que consideran aspectos metafísicos, que trascienden al saber físico o natural, como atributos de la ciencia (Ferrater Mora, 1994):

a) Ciencia como una entidad: Se emplea una metáfora ontológica (Lakoff y Johnson, 1980), para construir a la ciencia como una entidad poderosa de:

1. Saber:

"Sin la Ciencia no podríamos saber el por qué de las cosas [...]" (Gonzalo, Pachuca).

2. Poder:

"La Ciencia $[\ldots]$ hace milagros, logra hasta lo inimaginable e incluso alcanza fronteras que son todo menos accesibles [...] puede crear los elementos más magníficos [...] también puede destruirlos con gran facilidad [...] la ciencia es un arma de dos filos [...]" (Erika, Ciudad de México).

b) Significación religiosa católica

"Pero muchas personas se dejan llevar por las ideas religiosas que hay en lugares o culturas que creen en cosas inexplicables o milagros por así llamarles que no tienen explicación lógica y sería difícil de descifrar" (Sonia, Pachuca).

En este EDE la ciencia se instituye como una forma superior de conocimiento, que se construye mediante la crítica al misticismo (como revelación o iluminación) (Blauberg, 1975).

\section{Posturas actuales sobre la ciencia}

Aunque actualmente no existe consenso sobre las características que definen a la ciencia y al conocimiento científico, en este agrupamiento se incluyen EDE basados en posturas que hoy sostienen algunas comunidades científicas (Nieda y Macedo, 1997):

a) El conocimiento científico es relativo, desde dos diferentes puntos de vista:

1. El epistemológico

"Los científicos realizan actividades de diversa índole, según el marco científico donde se encuentren, ejemplo: Galileo Galilei se encontraba en el marco de la física y por ende, realizaba actividades de física [...]" (Fernando, Pachuca).

Entre los miembros de una comunidad científica se comparten las leyes y los supuestos teóricos que constituyen un paradigma (Chalmers, 1998).

2. El científico cultural

Cuando Erika (Ciudad de México) se refiere a la experimentación con animales, pregunta: Pero ¿acaso es adecuado comprender tanto? ¿¿A [sic] qué estamos dispuestos a hacer para lograrlo? [...] ¿la ciencia nos estará dando dominios que no nos corresponden?

La ciencia es una actividad impregnada por los valores e intereses particulares del momento histórico-social en el que se desarrolla (Nieda y Macedo, 1997).

b) Primero son los conocimientos previos, las ideas, la información, las hipótesis, la teoría

"Tales de Mileto que aportó la idea que la tierra era un disco plano que flotaba en el universo. Esta idea era equivocada pero aun así las ideas siguen aportando mucho a la ciencia” (Sonia, Jojutla).

Los nuevos conocimientos científicos se construyen a partir de los conocimientos previos sobre un tópico particular (Nieda y Macedo, 2008). 
c) Las teorías no son inmutables, se comprueban

Los científicos "realizan diferentes investigaciones para desarrollar hipótesis y comprobar teorías [...]" (Lucio, Jojutla). ${ }^{4}$

Este EDE sostiene que, una vez propuestas, las teorías especulativas han de ser probadas rigurosa e implacablemente por medio de la observación y la experimentación (Chalmers, 1998).

d) Preguntas y problemas

"La ciencia permite conocer la respuesta al por qué de las cosas [...]. Por qué [sic] llueve [...] por qué [sic] el relámpago $[\ldots]$ por qué $[s i c]$ el fuego" (Gonzalo, Pachuca). ${ }^{5}$

El punto de partida de la ciencia son los problemas sobre algún aspecto del mundo (Chalmers, 1998).

e) Diferentes metodologías

"Pienso que ese método se lo debe de plantear cada investigador y no tomar de guía absoluta a este método científico" (Marco, Jojutla).

La ciencia es una actividad con metodologías que no están sujetas a reglas fijas, por lo que no existe un método científico único y universal (Nieda y Macedo, 2008).

f) Reconstrucción del conocimiento

"La ciencia [...] es corregible y capaz de admitir mejoras. Esto es también gracias a los científicos, quienes no se conforman con una explicación brindada por sus antecesores, sino que se empeñan en conocer las cosas más y/o mejor, y al hacerlo, encuentran errores o mejoras" (Azucena, Ciudad de México).

Las teorías científicas se encuentran en constante revisión y reconstrucción (Nieda y Macedo, 2008). La ciencia progresa gracias al ensayo y al error, a las conjeturas y a las refutaciones (Chalmers, 1998).

\section{Confiabilidad de los datos}

Siguiendo el criterio de saturación para el control de la investigación (Callejo, 1998), la creación de nuevos grupos o categorías se suspendió cuando todos los EDE pudieron ser ubicados en una categoría ya definida. Como hemos apuntado, esta categorización no tiene pretensiones normativas o prescriptivas y, como puede apreciarse, tampoco exhaustivas. La agrupación que se presenta da cuenta de los datos aportados por los/as estudiantes que participaron en esta investigación.

Por otro lado, como se puede observar en el anexo, algunos EDE tienen una elevada cantidad de reiteraciones, tanto en el número de informantes que expresan el mismo sentido como el número de veces que un/a estudiante emplea el elemento en su argumentación. En concordancia con el mismo criterio de saturación, aquellos EDE que se encuentran en los textos de varios alumnos y alumnas son considerados válidos como elementos constitutivos de representaciones de la ciencia. No podría decirse lo mismo de aquellos EDE de los que solo se tiene un relato. Sin embargo, decidimos dejar en este informe la totalidad de los EDE para dar cuenta de la variedad de EDE constitutivos de RSC.

4. Con este ede los alumnos enfatizan la comprobación de las leyes o su refutación, cosa que no hacen otros elementos.

5. Con este EDE los informantes enfatizan los problemas o las preguntas como inicio del proceso de investigación, a diferencia del EDE que enfatiza la observación (ver en el agrupamiento "Los procesos"). 


\section{Elementos discursivo-epistemológicos en los textos individuales}

Diferentes EDE se entremezclan en cada texto, incluso, en un mismo fragmento de discurso:

Tabla 1.

Temas-segmentos en los que se divide el texto de Dora

(Ciudad de México) y elementos que constituyen su representación de la ciencia ${ }^{6}$

\begin{tabular}{|c|c|c|c|c|}
\hline \multirow{2}{*}{ Tema-segmento } & \multicolumn{4}{|c|}{ Elementos discursivo-epistemológicos } \\
\hline & La realidad & $\begin{array}{l}\text { Los procesos } \\
\text { empíricos }\end{array}$ & La ciencia es positiva & $\begin{array}{c}\text { Posturas actuales } \\
\text { sobre la ciencia }\end{array}$ \\
\hline Noción de ciencia & (1) & $\begin{array}{l}\text { Experiencia } \\
\text { sensorial (1) }\end{array}$ & $\begin{array}{l}\text { Conocimiento sistematizado (2) } \\
\text { Conocimiento verificado-objetivo (1) } \\
\text { Utilitarismo tecnológico (1) }\end{array}$ & \\
\hline $\begin{array}{l}\text { Origen y evolución } \\
\text { de la ciencia }\end{array}$ & & & $\begin{array}{l}\text { Acumulativa (2) } \\
\text { Conocimiento sistematizado (4) }\end{array}$ & \\
\hline $\begin{array}{l}\text { Metodología } \\
\text { científica }\end{array}$ & & $\begin{array}{l}\text { Experiencia (1) } \\
\text { Generalización } \\
\text { inductiva (1) }\end{array}$ & $\begin{array}{l}\text { Conocimiento verificado-objetivo (1) } \\
\text { Conocimiento sistematizado (1) } \\
\text { Método (2) } \\
\text { Acumulativa (1) }\end{array}$ & $\begin{array}{l}\text { Preguntas (2) } \\
\text { Relativismo } \\
\text { epistemológico (1) }\end{array}$ \\
\hline
\end{tabular}

Como muestra el ejemplo anterior, los elementos incluidos en el agrupamiento La ciencia es positiva recorren sintagmáticamente ${ }^{7}$ todo el discurso de Dora. El conocimiento organizado, sistematizado y especializado positivista, que participa en la argumentación de los tres temas en los que puede segmentarse su discurso, organiza la significación de todo el texto (Abric, 2001). Dora incluso recurre a la reiterada ejemplificación, ${ }^{8}$ en su afán por fijar este sentido en su representación de la ciencia. Por lo tanto, este EDE constituye el elemento central de la representación de la ciencia de Dora; alrededor de él se organizan otros elementos (Abric, 2001), como el conocimiento verificado-objetivo y la acumulación de conocimientos.

Algunos EDE se activan en la representación dependiendo del tema abordado (Guimelli, 2001). Por ejemplo, cuando Dora describe las características de la ciencia (su origen y evolución), considera que progresa mediante la acumulación de conocimientos sistematizados (La ciencia es positiva). En cambio, cuando argumenta sobre la metodología de investigación expresa, además de elementos de corte positivista, elementos relacionados con los procesos empíricos (participación de la experiencia y la generalización inductiva) y elementos que corresponden a las posturas actuales sobre la ciencia (participación de las preguntas en la investigación y el relativismo epistemológico) (tabla 1).

Lo anterior concuerda con lo expuesto por Ryder, Leach y Driver (1999), en cuanto a que los sujetos despliegan una imagen de la ciencia específica en un contexto particular y otra diferente en otro contexto. En este caso podemos hablar de contextos temático-discursivos diferentes.

De esta manera, para construir su representación de la ciencia los/as estudiantes activan ciertos elementos, y no otros, de una base común de EDE, denominada "fondo común de cultura" por Jodelete (1988). Probablemente, las representaciones expresadas por sujetos o grupos están condicionadas por

6. Se incluye el número de veces que se emplea cada elemento durante la argumentación, ya que la repetición o iteración es la redundancia o recurrencia semióticas que permiten fijar sentidos connotativos (Poloniato, 1998).

7. El sintagma es la combinación de signos lineal e irreversible, es la cadena hablada o discursiva; cada término adquiere su valor por semejanza o contraste a lo que precede y a lo que sigue. En el caso de los textos analizados, se consideran unidades sintagmáticas las oraciones, los párrafos o los segmentos discursivos.

8. El ejemplo fundamenta la regla que se está exponiendo (Perelman y Olbrechts-Tyteca, 1989). 
la accesibilidad a la información en torno al tema, por los intereses específicos y las preocupaciones particulares que varían según las inserciones sociales, así como por la experiencia en aspectos relacionados con el tema representado (Guimelli, 2001).

\section{Tendencias en la expresión de elementos discursivo-epistemológicos}

A pesar de la diversidad de EDE con los que los/as participantes en la investigación construyen sus representaciones de la ciencia, es posible identificar ciertas tendencias en su expresión discursiva. Destaca el predominio de elementos que se asemejan a posturas realistas, empirico-inductivistas (experiencia/ experimentación) y positivistas (conocimientos organizados/sistematizados, acumulación de conocimientos, utilitarismo tecnológico y conocimientos rigurosos-verdaderos). Tales elementos fueron expresados por la mayoría de los/as nueve estudiantes (anexo).

Estos resultados indican que existe una relación discursiva entre los elementos realistas, empiristas y positivistas. Existe también una relación epistemológica que podemos expresar de la siguiente manera: el mundo existe independientemente de nosotros (realismo), por lo que todo conocimiento deriva de la experiencia sensible (empirismo) y, así, mediante un procedimiento empirico-inductivo pueden obtenerse conocimientos objetivos-verdaderos (positivismo).

Este tipo de posturas empirico-positivistas también se encuentran presentes en diferentes mediaciones curriculares, así como en documentos de política educativa, programas de televisión educativa y prácticas de enseñanza de los/as docentes (Gordon, 1984). Probablemente estos elementos discursivoepistemológicos forman parte del núcleo central de la Rsc de las comunidades escolares a las que pertenecen el estudiantado. Estos elementos le confieren coherencia y estabilidad a la representación, por lo que esta evoluciona de manera muy lenta (Abric, 2001).

Sin embargo, las representaciones de la ciencia expresadas por los/as participantes también están conformadas por una variedad de EDE relacionados con las posturas actuales sobre la ciencia:

Tabla 2.

Frecuencia absoluta de elementos discursivo-epistemológicos empleados por la totalidad de los/as estudiantes participantes

\begin{tabular}{|l|c|c|c|c|c|}
\hline \multicolumn{1}{|c|}{ Elementos } & La realidad & $\begin{array}{c}\text { Los procesos } \\
\text { empíricos }\end{array}$ & $\begin{array}{c}\text { La ciencia es } \\
\text { positiva }\end{array}$ & $\begin{array}{c}\text { Elementos meta- } \\
\text { físicos }\end{array}$ & $\begin{array}{c}\text { Posturas actuales } \\
\text { sobre la ciencia }\end{array}$ \\
\hline Total & 27 & 67 & 138 & 21 & 66 \\
\hline
\end{tabular}

Aunque el eje central de las representaciones de la ciencia de la mayoría de los informantes está conformado por elementos positivistas, tres de ellos incorporan elementos de las posturas actuales sobre la ciencia en el sistema central o periférico de sus representaciones de la ciencia:

Tabla 3.

Número alumnos/as por postura epistemológica central y secundaria

\begin{tabular}{|c|l|}
\hline $\begin{array}{c}\text { Número } \\
\text { de alumnos y alumnas }\end{array}$ & \multicolumn{1}{c|}{ Postura } \\
\hline 5 & Positivista \\
\hline 2 & Positivista/Posturas actuales sobre la ciencia \\
\hline 1 & Positivista/Empírico-inductivista \\
\hline 1 & Posturas actuales sobre la ciencia/Empírico-inductivista \\
\hline
\end{tabular}




\section{CONCLUSIONES}

Los/as participantes en la investigación expresan en sus textos una amplia variedad de elementos discursivo-epistemológicos, que se agruparon a partir de sus similitudes y del parecido que guardan con posturas de corte realista, empirico-inductivista, positivista, metafísica y con las que actualmente sostienen algunas comunidades cientificas. Con estos EDE cada estudiante construye su particular representación de la ciencia.

Esta multiplicidad de posturas filosófico-epistemológicas y de estrategias discursivas con las que construyen probablemente se encuentre determinada por las diversas imágenes o representaciones de la ciencia a las que están expuestos los/as estudiantes a través de los medios de comunicación, de su contexto familiar, de sus prácticas de acercamiento a la ciencia, así como de los contenidos escolares y de las estrategias de enseñanza instrumentadas por sus profesores/as, entre otras posibles variables.

También es probable que, en la expresión individual de posturas epistemológicas, influyan las representaciones que cada estudiante se forma del contexto en el que trabaja (Ryder, Leach y Driver, 1999) (en este caso el Proyecto тAстісs), de la tarea que realiza durante su participación en la investigación que se reporta (reflexionar sobre "¿Qué es la ciencia? ¿Qué hacen y cómo trabajan los científicos?”), de la representación que tiene de sí mismo/a y de la que tiene de los otros/as participantes del proyecto (Abric, 2001). ${ }^{9}$

No es de extrañar, entonces, la presencia de posturas realistas en los discursos de los/as propios/as alumnos/as que han estado presentes a lo largo de la historia de las ideas filosóficas y pedagógicas desde la antigüedad (Abbagnano y Visalberghi, 2008). En la actualidad, estas posturas se encuentran en la escuela y forman parte del pensamiento de maestros/as de ciencias en formación, como lo demuestra un estudio en el que solo unos/as pocos/as consideran que las explicaciones teóricas no son reales, que son construcciones humanas que permiten explicar ciertos aspectos del mundo (Guisasola y Morentin, 2007).

Lo mismo sucede con otras ideas acerca de la naturaleza de la ciencia. A partir del trabajo de Bacon en el siglo XVII, por ejemplo, se consideró que el método empírico-inductivo proporcionaba un conocimiento cierto de las cosas. Si bien las personas que se dedican a la investigación científica observan, comparan, prueban, miden, especulan, conjeturan, construyen teorías y explicaciones, no existe una secuencia de actividades que permita resolver problemas científicos y proporcione conocimientos verdaderos (Lederman et al., 2002). Existe evidencia que sugiere que los libros de texto en uso en las escuelas secundarias presentan representaciones del método científico como una secuencia ordenada de pasos (Cordón, 2008).

Por otro lado, en el siglo xix, el positivismo acompaña y provoca el nacimiento y la afirmación de la organización técnico-industrial de la sociedad, que se pretende basada en el conocimiento científico, considerado como único saber auténtico. El positivismo expresa las esperanzas, los ideales y la exaltación optimista que acompañaron y provocaron esta fase del desarrollo de la sociedad. La finalidad última del positivismo de Comte era sustituir a la Iglesia católica por una Iglesia positiva, es decir, el orden basado en la voluntad divina por uno basado en las ciencias positivas (Abbagnano y Visalberghi, 2008). Este movimiento se da en un contexto sociohistórico, en que la Iglesia católica ya no podía explicar satisfactoriamente los fenómenos de la Naturaleza (Zea, 1985).

Diversos EDE, con los que los participantes en esta investigación construyen sus representaciones de la ciencia, guardan semejanza con las tesis de la filosofía positiva, introducida en diferentes ámbitos de la vida social en el siglo xix. Lo que no es de extrañar, pues esta aún continúa vigente en las aulas

9. Gonzalo, por ejemplo, concluye su texto de la siguiente manera: "Bye y escríbeme pronto. PD: soy Gonzalo" (Pachuca). Lo anterior demuestra las expectativas que este alumno genera al participar en el proyecto. 
de ciencias, forma parte del pensamiento del profesorado y de muchos libros de texto de uso cotidiano (Vázquez et al., 2012). Por lo tanto, esta poderosa influencia filosófico-epistemológica, que consideró a la ciencia como la base de un nuevo orden moral, religioso y político, subsiste hasta nuestros días y se expresa de diferentes maneras y de forma generalizada en los escritos de los/as estudiantes que participan en esta investigación.

A su vez, el constructivismo es una corriente epistemológica para la que el conocimiento es una construcción de la inteligencia humana, que va creando estructuras nuevas a partir de los conocimientos que se poseen (Mellado y Carracedo, 1993); mientras que el relativismo considera la ciencia una actividad para lograr conocimientos científicos - entre otras igualmente válidas-, que da importancia a los aspectos personales (intereses, creencias) y contextuales (sociales, políticos, económicos) en su generación y que sostiene que estos conocimientos no se construyen a partir exclusivamente de datos observacionales (Vázquez et al., 2012). Estos y otros paradigmas básicos sobre la $\mathrm{NdC}$ se encuentran en la base de las concepciones que sostienen algunas comunidades científicas en la actualidad y han sido retomados por las reformas curriculares, desde finales de los años ochenta y principios de los noventa del siglo xx, en respuesta a las necesidades educativas de muchos países.

$\mathrm{Si}$ bien estas corrientes de pensamiento sobre la ciencia se desarrollan bien entrado el siglo xx, ahora empiezan a tener una influencia significativa en las representaciones que sobre la ciencia construyen los/as alumnos/as de nivel medio superior. Probablemente, la expresión de estos elementos se encuentre vinculada con las características individuales y el contexto inmediato y contingente en el que se desenvuelven; lo que permite la adaptación y la diferenciación de las representaciones individuales mediante la integración de nueva información y lo vivido cotidianamente (Abric, 2001).

Se puede suponer así que los elementos que concuerdan con algunas posturas actuales sobre la ciencia presentes en los textos de Azucena (Ciudad de México), Lucio y Sonia (Jojutla), como parte del núcleo central o periférico de sus RSC, comienzan a formar parte del contexto social de las comunidades escolares a las que pertenecen, y de las que el Proyecto tactis es solo una muestra. Por medio de diferentes mediaciones institucionales (Quiroz, 1998), estos contenidos comienzan a instituirse como conocimientos legítimos (Edwards, 1999). Al parecer, la cultura dominante escolar ha incorporado estos elementos y favorece la expresión discursiva de representaciones de la ciencia sostenidas por algunas comunidades científicas actuales, lo que sugiere analizar con detalle la influencia que el trabajo colaborativo para la enseñanza de las ciencias y las reformas curriculares de corte constructivista están aportando para que ocurra lo anterior. Los resultados obtenidos también hablan de la conveniencia de dar mayor importancia a los contenidos sobre la naturaleza de la ciencia, en las prácticas y en los materiales didácticos.

Es así como después de considerar las representaciones de la ciencia que los jóvenes participantes en esta investigación han plasmado en sus escritos y de analizar los EDE con los que las construyen, puede observarse en primera instancia un hibridismo que dificulta categorizaciones precisas y fijas. Sin embargo, al historizar, considerar el proceso y contextualizar tal hibridismo, es posible ensayar una interpretación plausible de su trayecto. Lo que aparece como resultado de esta investigación es que las representaciones sociales se constituyen bajo condiciones socioculturales e históricas determinadas y que son susceptibles de cambio, siguiendo las modificaciones en esas condiciones. Se esboza entonces un proceso de transformación de representaciones sobre la ciencia, en algunos de los bachilleratos estudiados en esta investigación, similar al proceso de constitución y desarrollo de las ideas o teorías científicas propuesto por Toulmin (1977). 


\section{REFERENCIAS}

Abbagnano, N. y Visalberghi, A. (2008). Historia de la pedagogía. México: Fondo de Cultura Económica.

Abric, J. (2001). Las representaciones sociales: aspectos teóricos. En J. Abric. Prácticas sociales y representaciones. México: Ediciones Coyoacán.

Acevedo, J. (2008). El estado actual de la naturaleza de la ciencia en la didáctica de las ciencias. Revista Eureka sobre Enseñanza y Divulgación de la Ciencia, 5(2), pp. 134-169.

Aguirre, J. et al. (1990). Student-teachers' conceptions of science, teaching and learning: a case study in preservice science education. International Journal of Science Education, 12(4), pp. 381-390.

Ault, C. et al. (1984). Constructing Vee maps for clinical interviews on molecule concepts. Science Education, 68(4), pp. 441-463.

Barthes, R. (1992). Lo obvio y lo obtuso. Barcelona: Paidós.

Barthes, R. (1993). La aventura semiológica. Barcelona: Paidós.

Blauberg, I. (1975). Diccionario marxista de filosofía. México: Ediciones de Cultura Popular.

Buenfil, R. N. (1994). Cardenismo: Argumentación y Antagonismo en Educación. México: DIE (CINVESTAV)/CONACYT.

Buenfil, R. N. y Ruiz, M. M. (1997). Antagonismo y Articulación en el Discurso Educativo: Iglesia y Gobierno (1930-40 y 1970-93). México: Editorial Torres Asociados.

Buenfil, R. N. (2011). Apuntes sobre los usos de la teoría en la investigación social. Consideraciones metodológicas en investigación social. Saarbrucken: EAE-LAP LAMBERT Academic Publishing GmbH \& Co. KG.

Callejo, J. (1998). Los límites de la formalización de las prácticas cualitativas de investigación social: la saturación. Sociológica. Revista de pensamiento social, 3, pp. 93-119.

Chalmers, A. (1998). ¿Qué es esa cosa llamada ciencia? México: Siglo XXI.

Charaudeau, P. y Maingueneau, D. (2005). Diccionario de análisis del discurso. Buenos Aires: Amorrortu.

CORDón, R. (2008). Enseñanza y aprendizaje de procedimientos cientificos (contenidos procedimentales) en la educación secundaria obligatoria: Análisis de la situación, dificultades y perspectivas. Tesis doctoral. Universidad de Murcia. Disponible en: <http://digitum.um.es/xmlui/bitstream/10201/3613/1/ CordonAranda.pdf>. (Última consulta: 2 de abril de 2012).

Derrida, J. (1989). Márgenes de la Filosofía. Madrid: Cátedra.

Driver, R. et al. (1989). Ideas cientificas en la infancia y la adolescencia. Madrid: MEC/Morata.

Dogan, M. y Pahre, R. (1993). Las nuevas ciencias sociales. La marginalidad creadora. México: Grijalbo.

EDwArds, V. (1999). Las formas del conocimiento en el aula. En E. Rockwell (coord.). La escuela cotidiana. México: Fondo de Cultura Económica.

Ferrater Mora, J. (1994). Diccionario de Filosofía. Barcelona: Ariel.

FIfE-Schaw, CHR. (1993). Finding social representations in attribute checklists: how will we know when we have found one? En G. Breakwell y D. Canter (eds.). Empirical Approaches to Social Representations. Nueva York: Oxford University Press.

Gálvez, V. (2002). Las representaciones de la ciencia en la televisión educativa. El caso de la biología en Telesecundaria. Tesis de maestría 1995-2001 (CD). México: Departamento de Investigaciones Educativas (CINVESTAV).

Gálvez, V. y WaldegG, G. (2003). La negociación de significados asociados con la ciencia. Una metodología basada en ensayos individuales y la colaboración interpersonal. Revista Latinoamericana de Investigación Educativa, 3, vol. XXXIII, pp. 159-166. 
Gálvez, V. y Waldegg, G. (2004). Ciencia y cientificidad en la televisión educativa. Enseñanza de las Ciencias, 22(1), pp. 147-158.

GÁlvez, V. (2012). La escritura de textos en colaboración. Una práctica de construcción de representaciones sociales en el aprendizaje de las Ciencias. Revista Iberoamericana de Educación Superior, vol. 3, núm. 7.

Guisasola, J. y Morentin, M. (2007). ¿Comprenden la naturaleza de la ciencia los futuros maestros y maestras de Educación Primaria? Revista Electrónica de Enseñanza de las Ciencias, 6(2), pp. 246262.

Disponible en: <http://www.saum.uvigo.es/reec>. (Última consulta: 2 de abril de 2012).

Gordon, D. (1984). The Image of Science, Technological Consciousness, and the Hidden Curriculum. Curriculum Inquiry, 14(4), pp. 367-400.

Guimelli, CH. (2001). La función de enfermera. Prácticas y representaciones sociales. En J. C. Abric. Prácticas sociales y representaciones. México: Ediciones Coyoacán.

Haidar, J. (1996). El campo de la semiótica visual. De los sistemas a las prácticas semióticas. En A. Gimate y J. López (coord.). Semiótica. Memoria del curso 1995. México: Universidad Autónoma Metropolitana-Azcapotzalco.

Jodelet, D. (1988). La representación social: fenómenos, conceptos y teoría. En S. Moscovici. Psicología social, II. Pensamiento y vida social. Psicología y problemas sociales. Barcelona: Paidós.

Lakoff, G. y Johnson, M. (1980). Metáforas de la vida cotidiana. México: Cátedra.

Lederman, N. (1992). Students' and Teachers' Conceptions of the Nature of Sciencie: A Review of the Research. Journal of Research in Science Teaching, 29(4), pp. 331-359.

Lederman, N. et al. (2002). Views of Nature of Science Questionnaire: Toward Valid and Meaningful Assessment of Learners' Conceptions of Nature of Science. Journal of Research in Science Teaching, 39(6), pp. 497-521.

Lindeman, M. et al. (2002). Representing God. Papers on Social Representations, 11, pp. 1.1-1.13. Disponible en: <http://www.psr.jku.at>. (Última consulta: 2 de abril de 2012).

Marcuse, H. (2003). Razón y revolución. México: Alianza Editorial.

Mellado, V. y Carracedo, D. (1993). Contribuciones de la filosofía de la ciencia a la didáctica de las ciencias. Enseñanza de las Ciencias, 11(3), pp. 331-339.

Millar, R. y Driver, R. (1987). Beyond processes. Studies in Science Education, 14, pp. 33-62.

Moreno, L. y WaldegG, G. (1998). La epistemología constructivista y la didáctica de las ciencias: ¿coincidencia o complementariedad? Enseñanza de las ciencias, 16(3), pp. 421-429.

Moscovici, S. (1979). Psicoanálisis, su imagen y su público. Buenos Aires: Huemul.

Nascimento-Schulze, C. M. (1999). Social representation of the Universe. A Study with Doctors of Human and Natural Science. Papers on Social Representation, 8, pp. 5.1-5.13. Disponible en: $<$ http://www.psr.jku.at>. (Última consulta: 2 de abril de 2012).

Nieda, J. y Macedo, B. (1997). Un Currículo Cientifico para Estudiantes de 11 a 14 años. España: OEI-UNESCO/Santiago.

Newton, L. y Newton, D. (1998). Primary children's conceptions of science and the scientist: is the impact of a National Curriculum breaking down the stereotype? International Journal of Science Education, 20(9), pp. 1137-1149.

Novak, J. D. (1990). Concept mapping: A useful tool for science education. Journal of Research in Science Teaching, 27(10), pp. 937-949.

Poloniato, A. (1998). La lectura de los mensajes. Introducción al Análisis Semiótico de Mensajes. México: ILCE.

Quiroz, R. (1998). La reforma curricular de 1993 de la educación secundaria en México: nuevo currículum y prácticas de enseñanza. Investigación en la Escuela, 36, pp. 75-90. 
Ryder, J. et al. (1999). Undergraduate Science Students' Images of Science. Journal of Research in Science Teaching, 36 (2), pp. 201-219.

Southerland, S. y Gess-Newsome, J. (1999). Preservice Teachers' Views of Inclusive Science Teaching as Shaped by Images of Teaching, Learning, and Knowledge. Science Education, 83, pp. 131-150.

Toulmin, S. (1977). La comprensión humana. El uso colectivo y la evolución de los conceptos. Madrid: Alianza Universidad.

VÁzquez, A. et al. (s.f.). Cuatro paradigmas básicos sobre la naturaleza de la ciencia.

Disponible en: <http://www.oei.es/salactsi/acevedo20.htm>. (Última consulta: 2 abril de 2012).

WALDEGG, G. (2002). El uso de las nuevas tecnologías para la enseñanza y el aprendizaje de las ciencias. Revista Electrónica de Investigación Educativa, 4(1). Disponible en: <http://redie.ens.uabc.mx/ index.php/redie/article/view/53>. (Última consulta: 26 agosto de 2013).

Wilson, L. (1954). A study of opinions related to the nature of science and its purpose in society. Science Education, 38(4), pp. 236-242.

ZEA, L. (1985). El positivismo y la circunstancia mexicana. Lecturas Mexicanas, núm. 81. México: Fondo de Cultura Económica. 


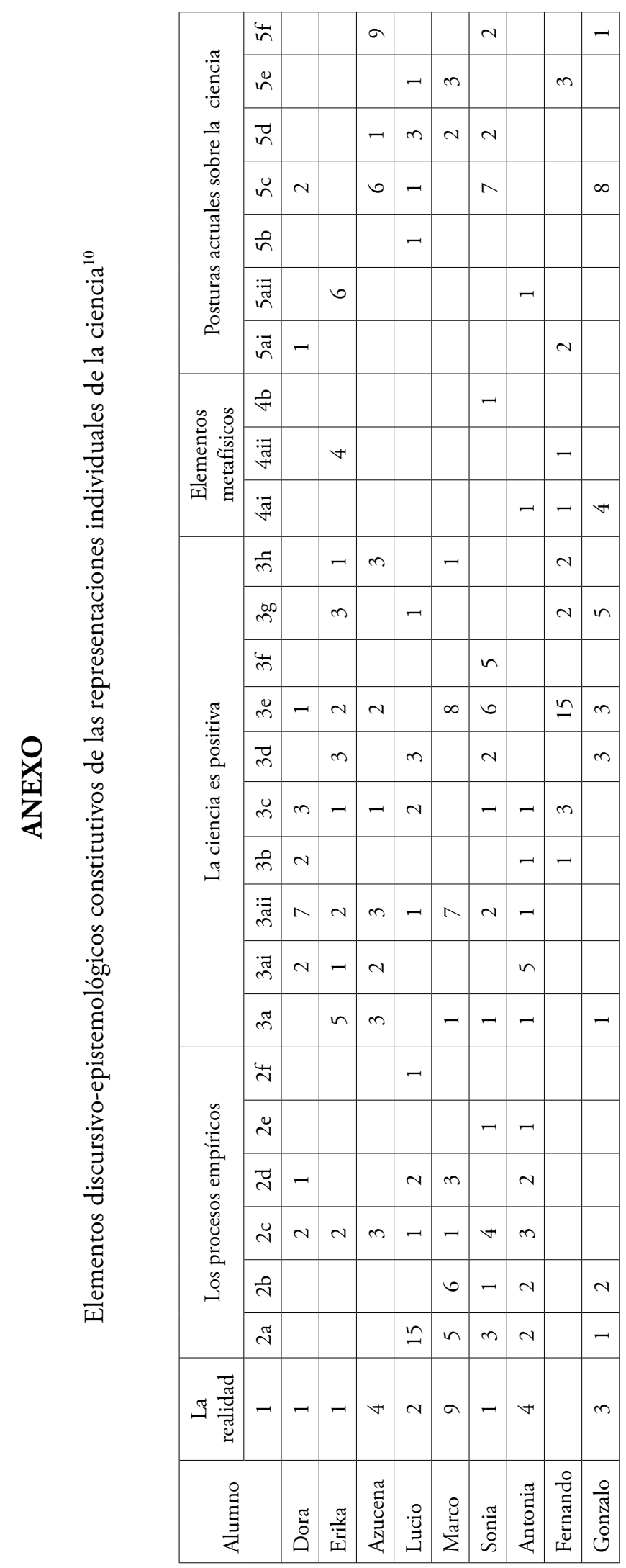




\title{
Science representations in writing compositions by mexican high school students
}

\author{
Víctor Gálvez Díaz \\ Secretaría de Educación Pública. Dirección General de Educación Tecnológica Industrial. México \\ galvezvictor@yahoo.com
}

\author{
Rosa Nidia Buenfil Burgos \\ Centro de Investigación y Estudios Avanzados. Departamento de Investigaciones Educativas. México \\ rbuenfil@cinvestav.mx
}

This paper describes the science representations as expressed in texts written by nine Mexican high school students aged between 16 and 18 years old, with different socio-cultural characteristics. Specifically, here will be described the discursive epistemological elements (DEE), as used by them in order to build their individual representations of science. The DEE's were grouped on the basis of the similarities between each other, and in accordance with some epistemological trends, such as realism, empiricism or positivism. This identification and subsequent grouping of the DEE has no normative or prescriptive intentions. In the context of this research, this task allowed to identify trends in the expression of the DEE among students participating in it and, at the same time, to suggest several possible interpretations.

The analysis of the representations of science acquires relevance, while the values and beliefs related to science and its development influence the activities of the individuals in contexts such as the school, where science is present daily.

A social representation of science (SRC) is considered in this research as a particular type of social representation that allows the individuals or groups to think and build knowledge about scientific topics that remain unknown for them. These representations are constructed based on shared knowledge within a community, in a given time.

In the school context, the SRC are particular constructions of reality; they are the result of different institutional mediations that are carried out through taking decisions and discrimination, which are based on what is conceived as validly knowable, since they define 'authoritatively' what the world is and, at the same time, the school is responsible for its transmission.

In the same way as semiotic constructivism does, in this research it is considered that the sign establishes a replacing symbolic relationship with things, so that the use of a word or an image has to evoke, allude, link with, or replace the thing in its absence. The language and the signs that form it incorporate the cultural conventions of a community and, at the same time, play a central role in the social construction of reality.

In a text, using examples, each participant was asked to describe what science means to him/her and how scientists work. Subsequently, we proceeded to apply the following analytical sequence:

a) Identification of the several DEE implied in each fragment of writing compositions.

b) Segmentation of each writing composition in accordance with the topics developed by each student.

c) Analysis of the way that signification of each item-segment of the writing text is organized by the DEE.

d) Identification of the manner in which the DEE organize the signification of the text as a whole, to form a particular representation of the science concept.

e) Grouping of the DEE by the similarities observed between them and their similarity with an epistemological trend.

The following is an example of an identified DEE in a student's text:

"[Science] is the study of everything that exists and its ways to manifest itself, its phenomena and causes". This DEE is related with the realistic trend, which considers that objects of reality have their own characteristics and they are independent of the ideas, beliefs or knowledge of individuals.

Beyond the diversity of DEE used by the participants in this research in order to form their science representations, it is possible to identify specific trends in its discursive expression. It stands out the predominance of elements that resemble realistic trends, empirical-inductivity trends (experience/experimentation) and positivist trends (organized/systematized knowledge, accumulation of knowledge, technological utilitarianism, rigorous/true knowledge). The majority of the nine students expressed such elements. However, the science representations expressed by the participants are also shaped by a variety of DEE related with the current stances about science.

This multiplicity of philosophical-epistemological positions and discursive strategies with which they build their science representations is probably modelled by the various images or representations of science to which they are exposed through the media, their family background and their practices to move closer to science, as well as the school contents and the teaching strategies implemented by their teachers, among other possible variables.

It is also likely that the individual expression of epistemological positions is influenced by representations that each student has conformed because of the working context, the task performed during their reported participation in the research (i.e. to reflect on "What is science? What do scientists do and how do they work?"), as well as the representation that everybody has of himself/herself and about the rest of the project participants. 